\title{
Symplectic Schemes for Linear Stochastic Schrödinger Equations with Variable Coefficients
}

\author{
Xiuling Yin ${ }^{1,2}$ and Yanqin Liu ${ }^{1,2}$ \\ ${ }^{1}$ School of Mathematical Sciences, Dezhou University, Dezhou 253023, China \\ ${ }^{2}$ The Center of Data Processing and Analyzing, Dezhou University, Dezhou 253023, China \\ Correspondence should be addressed to Xiuling Yin; yinxiuling@dzu.edu.cn
}

Received 16 December 2013; Accepted 4 April 2014; Published 22 April 2014

Academic Editor: Adem Kılıçman

Copyright (c) 2014 X. Yin and Y. Liu. This is an open access article distributed under the Creative Commons Attribution License, which permits unrestricted use, distribution, and reproduction in any medium, provided the original work is properly cited.

\begin{abstract}
This paper proposes a kind of symplectic schemes for linear Schrödinger equations with variable coefficients and a stochastic perturbation term by using compact schemes in space. The numerical stability property of the schemes is analyzed. The schemes preserve a discrete charge conservation law. They also follow a discrete energy transforming formula. The numerical experiments verify our analysis.
\end{abstract}

\section{Introduction}

Differential equations (DEs) are important models in sciences and engineering. By theoretical and numerical analysis of DEs, we can yield some mathematical explanation of many phenomena in applied sciences [1-5]. Time-dependent Schrödinger equations arise in quantum physics, optics, and many other fields $[6,7]$. Some numerical methods for such equations, such as symplectic scheme and multisymplectic schemes, have been proposed in [8-14]. The schemes possess good numerical stability. Compact schemes are popular recently due to high accuracy, compactness, and economic resource in scientific computation [15-17]. In this paper, applying compact operators, we construct symplectic methods to the initial boundary problems of the linear Schrödinger equation with a variable coefficient and a stochastic perturbation term (denoted by LSES):

$$
\begin{gathered}
i u_{t}+u_{x^{4}}+f(x) u=\epsilon u \circ \dot{\chi}, \quad x \in[0, L], \\
u(x, 0)=u_{0}(x), \quad t \in[0, T], \\
u(0, t)=u(L, t),
\end{gathered}
$$

where $i^{2}=-1, f(x)$ is a real differential function, $u_{0}(x)$ is a differential function, $\epsilon$ is a small real number, and o means
Stratonovich product. $\dot{\chi}$ is a real-valued white noise which is delta correlated in time and either smooth or delta correlated in space. For an integer $m, u_{x^{m}}$ and $u_{t^{m}}$ mean the $m$-order partial derivatives of $u$ with respect to $x$ and $t$, respectively. The system (1) with $\epsilon=0$ is a deterministic system. When $\epsilon$ is small, we can think that (1) is perturbed by the stochastic term.

By multiplying (1) by $\bar{u}$ or $\bar{u}_{t}$ and then integrating it with respect to $t$ and $x$, it is easy to verify the following result.

Proposition 1. Under the periodic boundary condition,

(a) the solution of (1) satisfies the charge conservation law

$$
\mathscr{Q}(t)=\int_{0}^{L}|u(x, t)|^{2} d x=\mathscr{Q}(0),
$$

(b) the corresponding deterministic system $(\epsilon=0)$ of (1) possesses the energy conservation law

$$
\mathscr{E}(t)=\int_{0}^{L}\left|u_{x x}\right|^{2}+f(x)|u|^{2} d x=\mathscr{E}(0) .
$$

The paper is organized as follows. In Section 2, we give a symplectic structure of the LSES. In Section 3, we present the 
new symplectic methods to the LSES. First, we use a kind of compact schemes in discretization of spatial derivative. Then, in temporal discretization, we adopt the symplectic midpoint method. The new methods are denoted by LSC schemes. We also analyze the numerical stability of LSC schemes. We give two numerical examples to support our theory in Section 4. At last, we make some conclusions.

\section{Symplectic Structure of the LSES}

Let $u=p+i q$. The LSES (1) can be written in

$$
\begin{gathered}
p_{t}+q_{x^{4}}+f(x) q=\epsilon q \circ \dot{\chi}, \\
-q_{t}+p_{x^{4}}+f(x) p=\epsilon p \circ \dot{\chi} .
\end{gathered}
$$

Introducing the variable $z=(p, q)^{T},(4)$ reads in stochastic symplectic context

$$
z_{t}=J^{-1} \nabla_{z} H(z)+\epsilon J^{-1} \nabla_{z} S(z) \circ \dot{\chi}
$$

where

$$
\begin{gathered}
H(z)=\frac{1}{2}\left(p_{x x}^{2}+q_{x x}^{2}\right)+\frac{f(x)}{2}\left(p^{2}+q^{2}\right), \\
S(z)=-\frac{1}{2}\left(p^{2}+q^{2}\right), \\
J=\left(\begin{array}{cc}
0 & 1 \\
-1 & 0
\end{array}\right) .
\end{gathered}
$$

The system satisfies the symplectic conservation law $[7,12$, 18]:

$$
\omega_{t}=0, \quad \omega=d p \wedge d q .
$$

Numerical methods which preserve the discrete symplectic conservation law are called symplectic methods. Symplectic methods have good numerical stability.

\section{LSC Schemes}

3.1. Compact Scheme. Introduce the following uniform mesh grids:

$$
x_{k}=k h, \quad k=0,1, \ldots, N ; \quad t_{n}=n \tau, \quad n=0,1, \ldots,
$$

where $h=L / N$ and $\tau$ are spatial and temporal step sizes, respectively. Denote the numerical values of $u\left(x_{k}, t_{n}\right)$ at the nodes $\left(x_{k}, t_{n}\right)$ by $u_{k}^{n}$. The symbols $u^{n}$ and $u_{k}$ mean the numerical solution vectors at $t=t_{n}$ and $x=x_{k}$ with components $u_{k}^{n}$, respectively. Furthermore, we denote

$$
u_{k}^{n+(1 / 2)}:=\frac{u_{k}^{n+1}+u_{k}^{n}}{2}, \quad \delta_{t} u_{k}^{n+(1 / 2)}:=\frac{u_{k}^{n+1}-u_{k}^{n}}{\tau} .
$$

Introducing the following linear operators

$$
\begin{gathered}
\mathscr{A} u_{k}=\alpha u_{k-1}+u_{k}+\alpha u_{k+1}, \\
\mathscr{B} u_{k}=b \frac{u_{k+3}-9 u_{k+1}+16 u_{k}-9 u_{k-1}+u_{k-3}}{6 h^{4}} \\
+a \frac{u_{k+2}-4 u_{k+1}+6 u_{k}-4 u_{k-1}+u_{k-2}}{h^{4}},
\end{gathered}
$$

we adopt formula [19]

$$
\delta_{x}^{4} u_{k}=\mathscr{A}^{-1} \mathscr{B} u_{k}
$$

to approximate $u_{x^{4}}$, which means that

$$
\mathscr{A} \delta_{x}^{4} u_{k}=\mathscr{B} u_{k}
$$

By Taylor expansion, we can derive a family of fourth-order schemes with

$$
a=2(1-\alpha), \quad b=4 \alpha-1 .
$$

The leading term of the truncation error of the method is ((7$26 \alpha) / 240)\left(u_{x^{8}}\right)_{k} h^{4}$. If $b=0$, we get a scheme with smaller stencil. A sixth-order scheme is obtained with

$$
\alpha=\frac{7}{26}, \quad a=\frac{19}{13}, \quad b=\frac{1}{13} .
$$

Denote two symmetric and cyclic matrices by

$$
A=\left[\begin{array}{ccccccc}
1 & \alpha & 0 & \cdots & \cdots & 0 & \alpha \\
\alpha & 1 & \alpha & 0 & \cdots & \cdots & 0 \\
0 & \alpha & 1 & \alpha & 0 & \cdots & 0 \\
\vdots & \ddots & \ddots & \ddots & \ddots & \ddots & \vdots \\
\vdots & \vdots & \ddots & \alpha & 1 & \alpha & 0 \\
0 & 0 & \cdots & 0 & \alpha & 1 & \alpha \\
\alpha & 0 & \cdots & \cdots & 0 & \alpha & 1
\end{array}\right]_{N \times N}
$$




$$
B=\frac{1}{6 h^{4}}\left[\begin{array}{ccccccccccc}
c_{0} & c_{1} & 6 a & b & 0 & \cdots & \cdots & 0 & b & 6 a & c_{1} \\
c_{1} & c_{0} & c_{1} & 6 a & b & 0 & \cdots & \cdots & 0 & b & 6 a \\
6 a & c_{1} & c_{0} & c_{1} & 6 a & b & 0 & \cdots & \cdots & 0 & b \\
b & 6 a & c_{1} & c_{0} & c_{1} & 6 a & b & 0 & \cdots & \cdots & 0 \\
0 & b & 6 a & c_{1} & c_{0} & c_{1} & 6 a & b & 0 & \cdots & 0 \\
\vdots & \ddots & \ddots & \ddots & \ddots & \ddots & \ddots & \ddots & \ddots & \ddots & \vdots \\
0 & \cdots & 0 & b & 6 a & c_{1} & c_{0} & c_{1} & 6 a & b & 0 \\
0 & \cdots & \cdots & 0 & b & 6 a & c_{1} & c_{0} & c_{1} & 6 a & b \\
b & 0 & \cdots & \cdots & 0 & b & 6 a & c_{1} & c_{0} & c_{1} & 6 a \\
6 a & b & 0 & \cdots & \cdots & 0 & b & 6 a & c_{1} & c_{0} & c_{1} \\
c_{1} & 6 a & b & 0 & \cdots & \cdots & 0 & b & 6 a & c_{1} & c_{0}
\end{array}\right]_{N \times N}
$$

where $c_{0}=16 b+36 a$ and $c_{1}=-9 b-24 a$. Then the matrix form of (11) is

$$
\delta_{x}^{4} u^{n}=A^{-1} B u^{n}
$$

3.2. Discretization of the LSES. Applying the approximation (11) to linear system (4), we obtain the following semidiscretization stochastic Hamiltonian system:

$$
\left(z_{k}\right)_{t}=J^{-1} \nabla_{z} \bar{H}\left(z_{k}\right)+\epsilon J^{-1} \nabla_{z} \bar{S}\left(z_{k}\right) \circ \dot{\chi}_{k},
$$

where

$$
\begin{gathered}
\bar{H}(z)=\frac{f\left(x_{k}\right)}{2}\left(p_{k}^{2}+q_{k}^{2}\right)+\frac{1}{2} \delta_{x}^{4}\left(p_{k}^{2}+q_{k}^{2}\right) \\
\bar{S}(z)=-\frac{1}{2}\left(p_{k}^{2}+q_{k}^{2}\right) .
\end{gathered}
$$

In temporal discretization of (17), we apply the symplectic midpoint method

$$
\begin{aligned}
\delta_{t} z_{k}^{n+(1 / 2)}= & J^{-1} \nabla_{z} \bar{H}\left(z_{k}^{n+(1 / 2)}\right)+\epsilon J^{-1} \nabla_{z} \bar{H}\left(z_{k}^{n+(1 / 2)}\right) \\
& \circ \dot{\chi}_{k}^{n+(1 / 2)} .
\end{aligned}
$$

Its componentwise formulation is

$$
\begin{aligned}
\frac{p_{k}^{n+1}-p_{k}^{n}}{\tau}= & -f\left(x_{k}\right) q_{k}^{n+(1 / 2)}-\delta_{x}^{4} q_{k}^{n+(1 / 2)} \\
& +\epsilon q_{k}^{n+(1 / 2)} \circ \dot{\chi}_{k}^{n+(1 / 2)}, \\
\frac{q_{k}^{n+1}-q_{k}^{n}}{\tau}= & f\left(x_{k}\right) p_{k}^{n+(1 / 2)}+\delta_{x}^{4} p_{k}^{n+(1 / 2)} \\
& -\epsilon p_{k}^{n+(1 / 2)} \circ \dot{\chi}_{k}^{n+(1 / 2)} .
\end{aligned}
$$

According to the Fourier analysis, the LSC schemes (19) are unconditionally stable. In fact, we can derive

$$
\delta_{x}^{4} z_{k}^{n}=\mu z_{k}^{n}
$$

where

$$
\mu=\frac{4(\omega-1)^{2}(3 a+2 b+b \omega)}{3 h^{4}(1+2 \alpha \omega)}, \quad \omega=\cos \beta h .
$$

Then, with (19) and (21), we can obtain $z^{n+1}=G z^{n}$ with

$$
\left(1+\frac{c^{2} \tau^{2}}{4}\right) G=\left(\begin{array}{cc}
1-\frac{c^{2} \tau^{2}}{4} & -c \tau \\
c \tau & 1-\frac{c^{2} \tau^{2}}{4}
\end{array}\right)
$$

where $c=\mu+f\left(x_{k}\right)-\epsilon \circ \dot{\chi}_{k}^{n+(1 / 2)}$. By direct computation, we can derive that the spectral radius of the matrix $G$ is 1 and $\|G\|_{2}=1$. Therefore, the scheme (19) is unconditionally stable. Moreover, by symmetry, they are nondissipative.

Theorem 2. Let $\left\|z^{n}\right\|^{2}=h \sum_{k} z_{k}^{n} \overline{z_{k}^{n}}$. Then, $\left\|z^{n}\right\|$ is the discrete charge invariant of the LSC schemes (19), which implies the discrete charge conservation law of (2).

Scheme (19) can be rewritten in compact form

$$
\begin{aligned}
i \mathscr{A} & \frac{u_{k}^{n+1}-u_{k}^{n}}{\tau}+\mathscr{B} u_{k}^{n+(1 / 2)}+\mathscr{A} f\left(x_{k}\right) u_{k}^{n+(1 / 2)} \\
=\epsilon \mathscr{A} u_{k}^{n+(1 / 2)} \circ \dot{\chi}_{k}^{n+(1 / 2)} &
\end{aligned}
$$

Multiplying (24) by $\left(\overline{u_{k}^{n+1}}-\overline{u_{k}^{n}}\right)$ and summing over $k$, we obtain

$$
\begin{aligned}
\frac{i \tau}{2} \sum_{k}\left[\mathscr{B} u_{k}^{n+1} \overline{u_{k}^{n+1}}-\mathscr{B} u_{k}^{n} \overline{u_{k}^{n}}\right] \\
+\frac{i \tau}{2} \sum_{k} \mathscr{A} f\left(x_{k}\right)\left[u_{k}^{n+1} \overline{u_{k}^{n+1}}-u_{k}^{n} \overline{u_{k}^{n}}\right] \\
\quad-\frac{i \tau \epsilon}{2} \sum_{k}\left[\mathscr{A} u_{k}^{n+1} \circ \dot{\chi}_{k}^{n+(1 / 2)} \overline{u_{k}^{n+1}}-\mathscr{A} u_{k}^{n} \circ \dot{\chi}_{k}^{n+(1 / 2)} \overline{u_{k}^{n}}\right] \\
+\frac{i \tau}{2} \sum_{k}\left[\mathscr{B} u_{k}^{n} \overline{u_{k}^{n+1}}-\mathscr{B} u_{k}^{n+1} \overline{u_{k}^{n}}\right] \\
+\frac{i \tau}{2} \sum_{k} \mathscr{A} f\left(x_{k}\right)\left[u_{k}^{n} \overline{u_{k}^{n+1}}-u_{k}^{n+1} \overline{u_{k}^{n}}\right] \\
\quad-\frac{i \tau \epsilon}{2} \sum_{k}\left[\mathscr{A} u_{k}^{n} \circ \dot{\chi}_{k}^{n+(1 / 2)} \overline{u_{k}^{n+1}}-\mathscr{A} u_{k}^{n+1} \circ \dot{\chi}_{k}^{n+(1 / 2)} \overline{u_{k}^{n}}\right] \\
=\sum_{k}\left[\mathscr{A}\left(u_{k}^{n+1}-u_{k}^{n}\right)\left(\overline{u_{k}^{n+1}}-\overline{u_{k}^{n}}\right)\right] .
\end{aligned}
$$




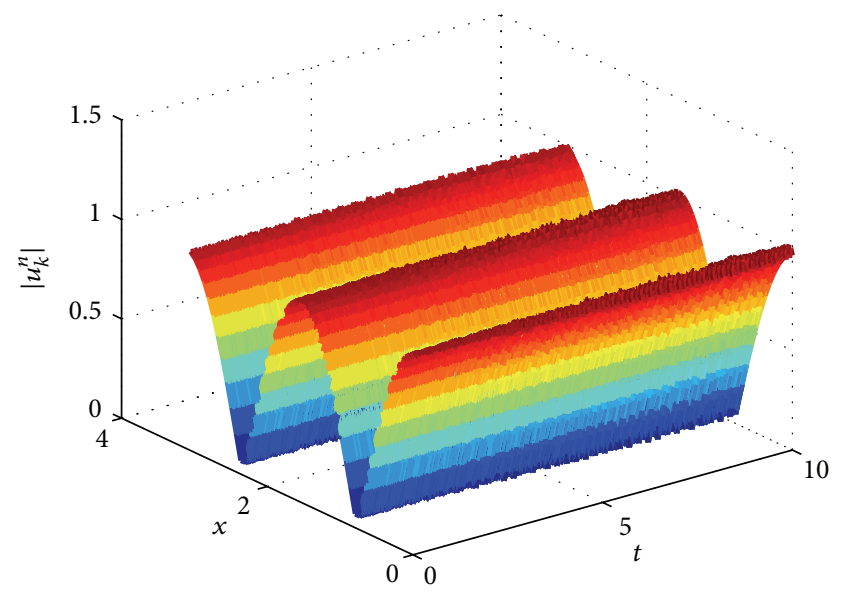

(a) $\epsilon=0.05$

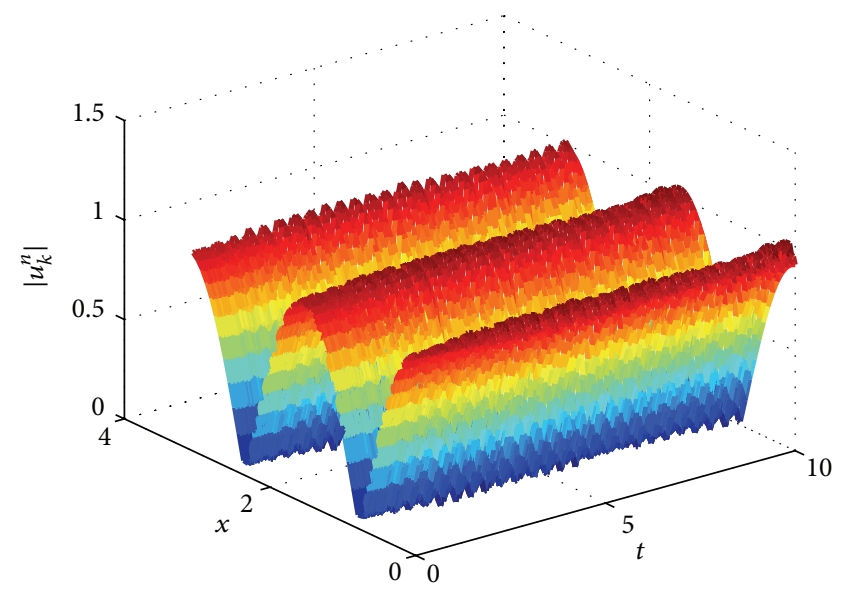

(b) $\epsilon=0.1$

FIGURE 1: $\left|u_{k}^{n}\right|$ for one trajectory.

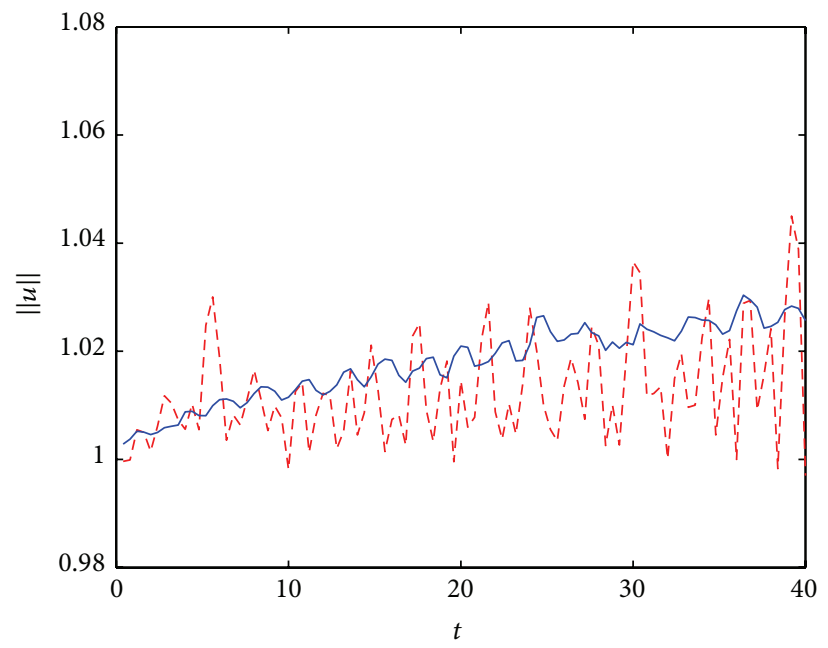

(a)

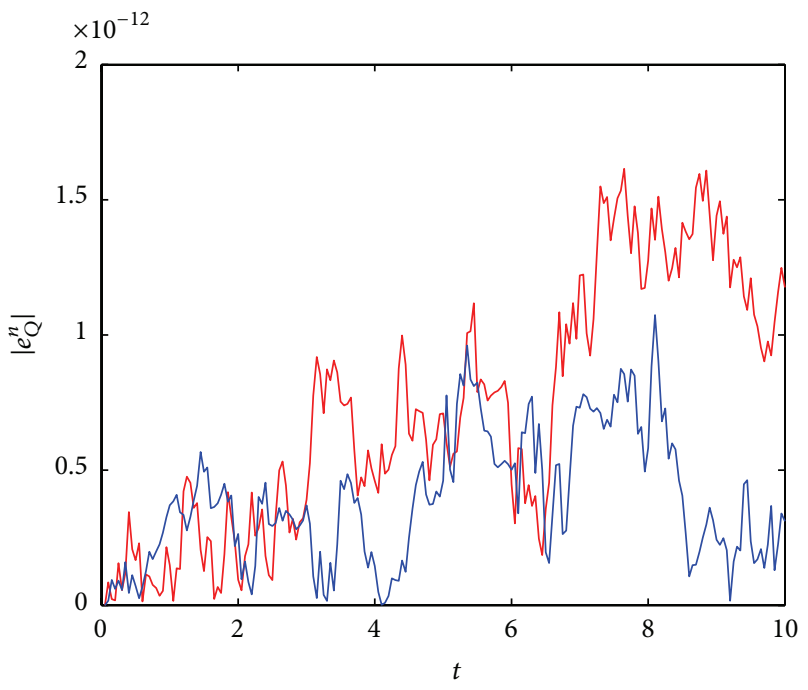

(b)

FIGURE 2: $\|u\|_{\infty}$ for one trajectory and the average norm over 50 trajectories (a). Residuals of discrete charge for one trajectory (b).

Since $\mathscr{A}$ and $\mathscr{B}$ are symmetric, the first three summation terms in the above equality are purely imaginary, while the last four summation terms are real. Denote

$$
\begin{aligned}
E^{n}= & h \sum_{k}\left[\mathscr{B} u_{k}^{n} \overline{u_{k}^{n}}\right]+h \sum_{k}\left[\mathscr{A} f\left(x_{k}\right) u_{k}^{n} \overline{u_{k}^{n}}\right] \\
& -\frac{h}{2} \epsilon \sum_{k}\left[\mathscr{A} u_{k}^{n} \circ \dot{\chi}_{k}^{n} \overline{u_{k}^{n}}\right] .
\end{aligned}
$$

Now, taking the imaginary parts of (25), we can get that

$$
E^{n+1}-E^{n}=\frac{h}{2} \epsilon \sum_{k}\left[\mathscr{A} u_{k}^{n+1} \circ \dot{\chi}_{k}^{n} \overline{u_{k}^{n+1}}-\mathscr{A} u_{k}^{n} \circ \dot{\chi}_{k}^{n+1} \overline{u_{k}^{n}}\right] .
$$

Denote

$$
\begin{gathered}
v_{k}^{n}=\sqrt{\frac{b}{6} \frac{u_{k+3}^{n}-u_{k}^{n}}{h^{2}}}, \quad \widetilde{v}_{k}^{n}=\sqrt{\frac{3 b}{2}} \frac{u_{k+1}^{n}-u_{k}^{n}}{h^{2}}, \\
w_{k}^{n}=\sqrt{a} \frac{u_{k+2}^{n}-u_{k}^{n}}{h^{2}}, \\
\widetilde{w}_{k}^{n}=2 \sqrt{a} \frac{u_{k+1}^{n}-u_{k}^{n}}{h^{2}}, \quad y_{k}^{n}=\mathscr{A} f\left(x_{k}\right) u_{k}^{n}, \\
\widetilde{y}_{k}^{n}=\mathscr{A} u_{k}^{n} \circ \dot{\chi}_{k}^{n}, \\
\left\langle u^{n}, y^{n}\right\rangle=h \sum_{k} u_{k}^{n} y_{k}^{n}, \quad\left\|u^{n}\right\|^{2}=\left\langle u^{n}, u^{n}\right\rangle .
\end{gathered}
$$




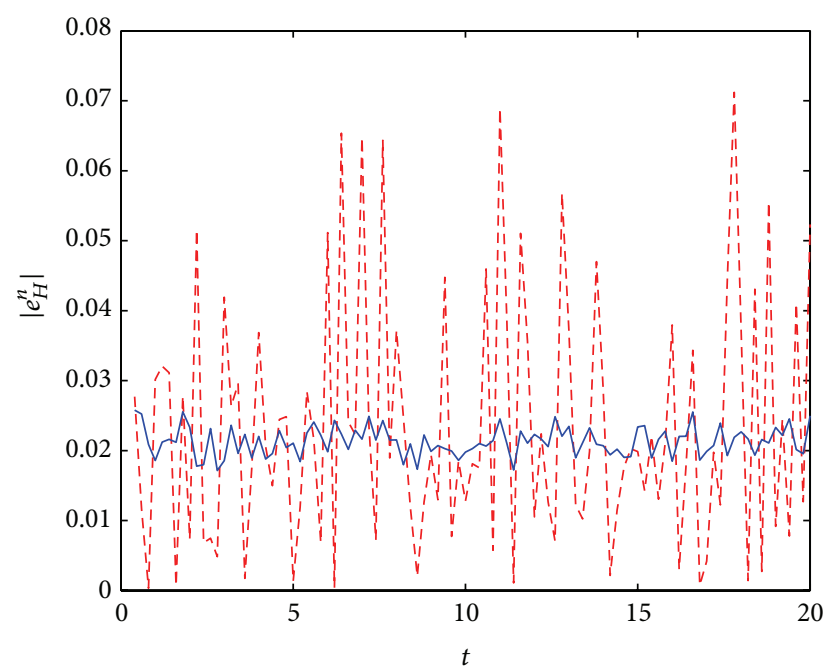

(a) $e_{H}^{n}$

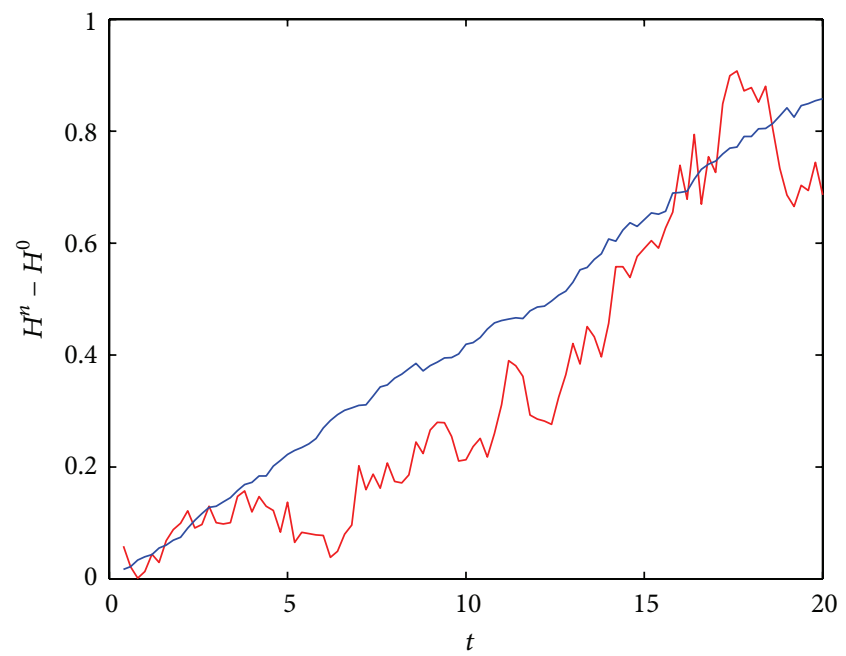

(b) $H^{n}-H^{0}$

FIGURE 3: Residuals of discrete energy for one trajectory and the average energy over 50 trajectories.

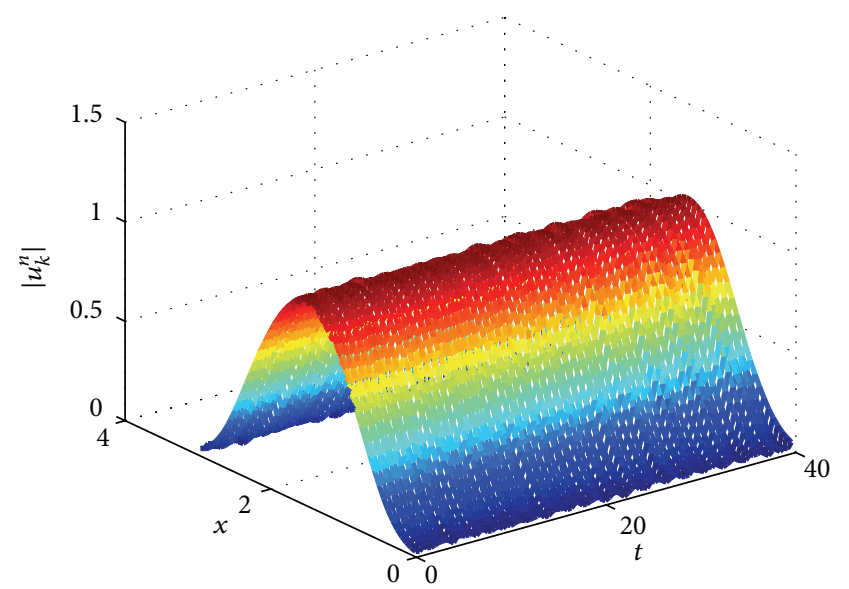

(a) $\epsilon=0.05$

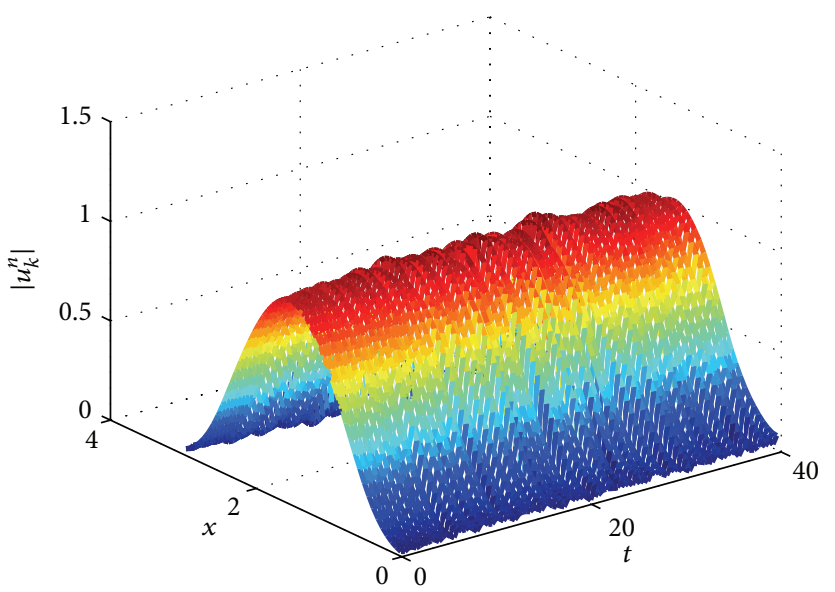

(b) $\epsilon=0.1$

FIgURE $4:\left|u_{k}^{n}\right|$ for one trajectory.

According to the Green formula, we obtain that

$$
h \sum_{k}\left[\mathscr{B} u_{k}^{n} \overline{u_{k}^{n}}\right]=\left\|\widetilde{v}^{n}\right\|^{2}-\left\|v^{n}\right\|^{2}+\left\|\widetilde{w}^{n}\right\|^{2}-\left\|w^{n}\right\|^{2} .
$$

Then,

$$
\begin{aligned}
E^{n}= & \left\|\widetilde{v}^{n}\right\|^{2}-\left\|v^{n}\right\|^{2}+\left\|\widetilde{w}^{n}\right\|^{2}-\left\|w^{n}\right\|^{2} \\
& +\left\langle y^{n}, u^{n}\right\rangle-\frac{\epsilon}{2}\left\langle\tilde{y}^{n}, u^{n}\right\rangle .
\end{aligned}
$$

Therefore, from the above analysis, we give the following result.

Theorem 3. Under the periodic boundary condition, the LSC schemes (19) satisfy the discrete energy transforming law (27).

\section{Numerical Examples}

We use the LSC scheme to solve the LSESs and investigate its numerical behavior. According to the precise mathematical definition of the white noise $[13,14]$, we can simulate the noise as $\dot{\chi}_{k}^{n+(1 / 2)}=(1 / \sqrt{h \tau}) \chi_{k}^{n+(1 / 2)}$, where $\chi_{k}^{n+(1 / 2)}, k=0,1, \ldots, N$ is a sequence of independent random variables with normal law $\mathcal{N}(0,1)$ at each time increment. Denote

$$
\begin{gathered}
\left\|u^{n}\right\|_{\infty}=\max _{1 \leq k \leq N}\left|u_{k}^{n}\right|, \quad e_{\mathrm{Q}}^{n}=Q^{n}-Q^{0}, \\
e_{H}^{n}=H^{n}-H^{n-1} .
\end{gathered}
$$

The numerical residuals of $\mathscr{Q}(t)$ and $\mathscr{H}(t)$ are measured by $e_{\mathrm{Q}}^{n}$ and $e_{H}^{n}$, respectively. For numerical computation, we take $\tau=0.01, h=\pi / 50$, and $\epsilon=0.05,0.1$. 


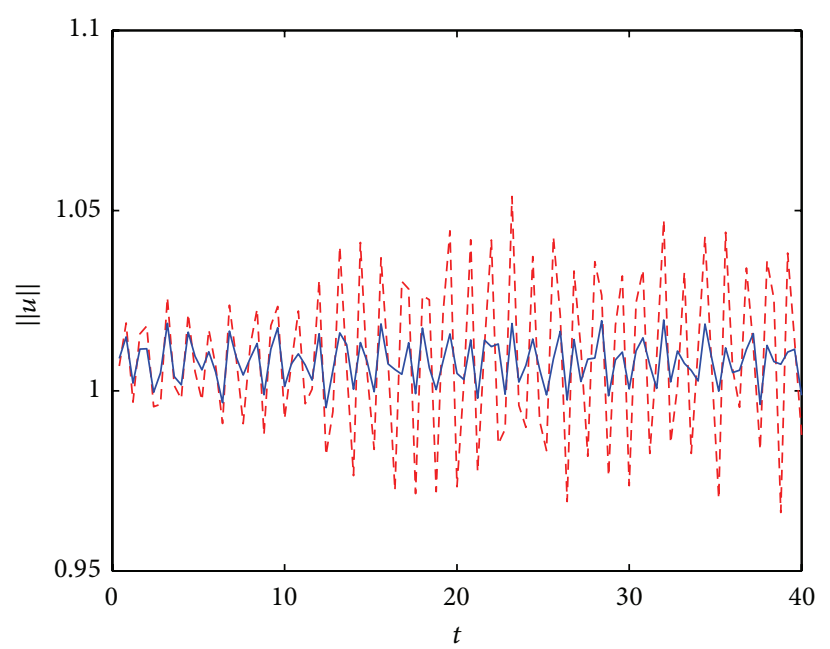

(a)

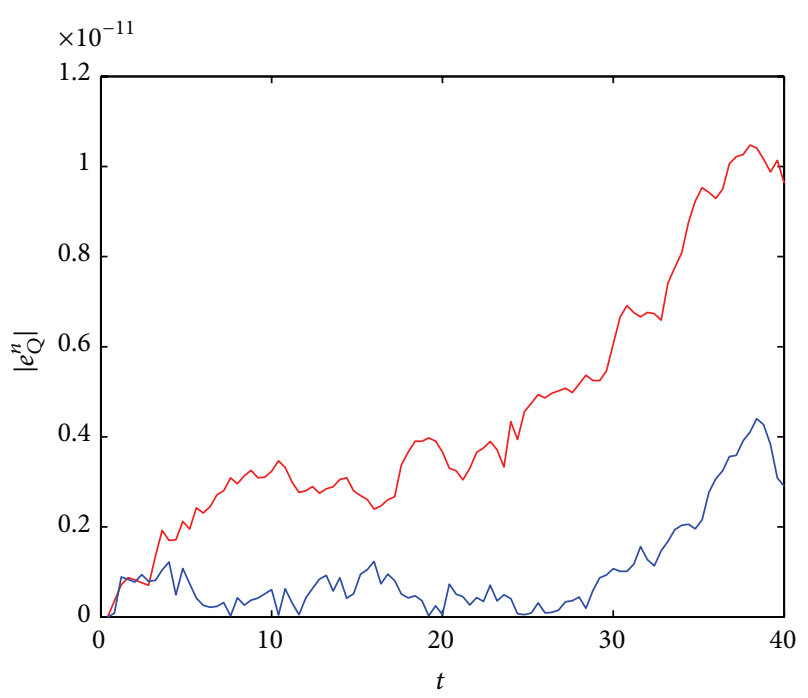

(b)

FIGURE 5: $\|u\|_{\infty}$ for one trajectory and the average norm over 50 trajectories (a). Residuals of discrete charge for one trajectory (b).

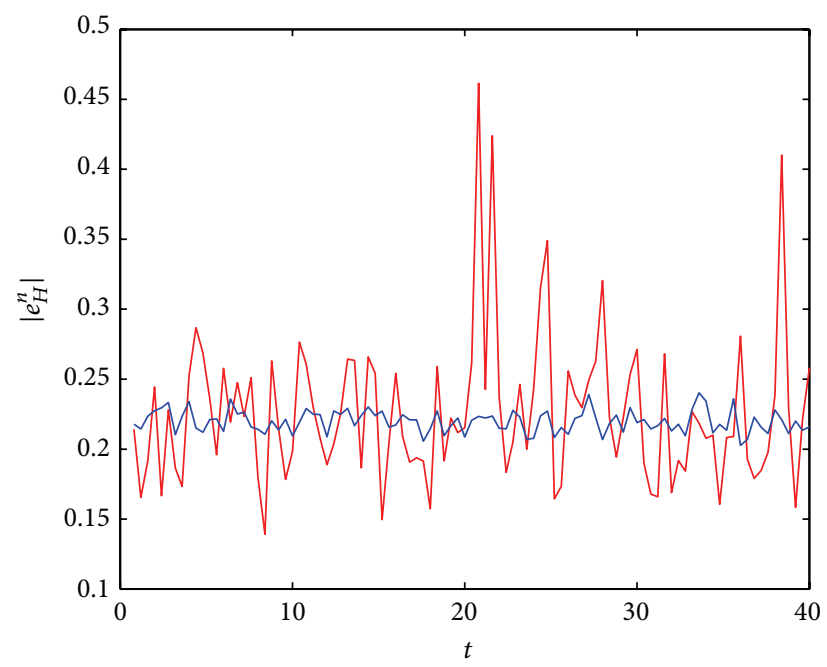

(a) $e_{H}^{n}$

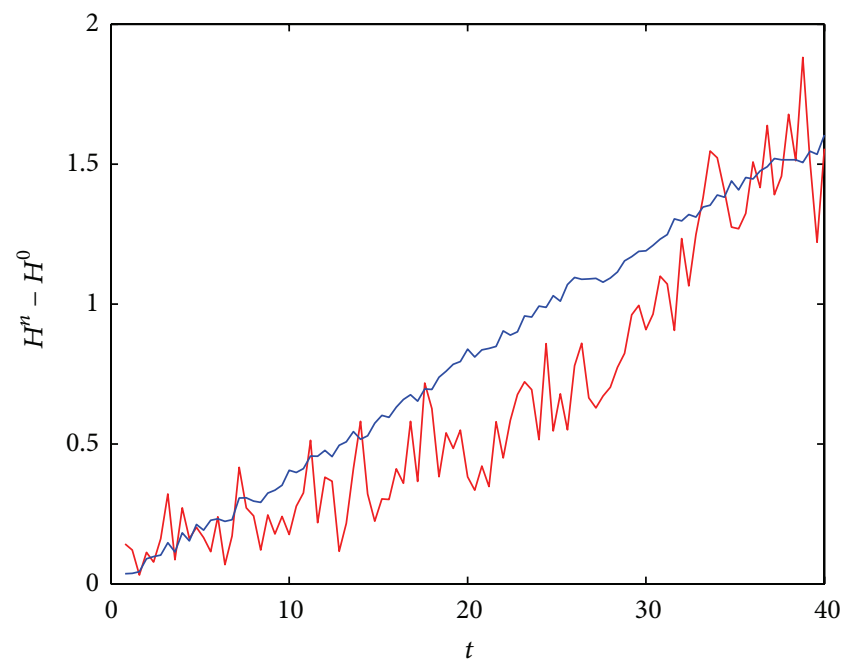

(b) $H^{n}-H^{0}$

FIGURE 6: Residuals of discrete energy for one trajectory and the average energy over 50 trajectories.

Example 1. LSE with constant coefficients and periodic boundary condition.

Consider

$$
\begin{gathered}
i u_{t}+u_{x^{4}}-15 u=\epsilon \mathcal{u} \circ \dot{\chi}, \quad x \in[0, \pi], t>0, \\
u(x, 0)=\exp \left(\frac{i \pi}{3}\right) \cos 2 x .
\end{gathered}
$$

The exact solution of its deterministic system is $u(x, t)=$ $\exp [i(t+(\pi / 3))] \cos 2 x$. The right side in the above system can be seen as a stochastic perturbation term.

Figure 1 plots the amplitude $\left|u_{k}^{n}\right|$ for one trajectory. Figure 2 shows the evolution of $\|u\|_{\infty}$ for one trajectory and the average norm over 50 trajectories. We see that the white noise produces stochastic perturbation on the solitary wave and the size of perturbation depends on the size of noise. Figure 2 plots the residuals of discrete charge of one trajectory, which verifies the conservation of discrete charge of the compact schemes. Figure 3 plots the residuals of discrete energy for one trajectory and the average norm over 50 trajectories. The figure tells us that the stochastic noise makes residuals of discrete energy increase linearly over time.

Example 2. LSE with a variable coefficient and periodic boundary condition.

Consider

$$
\begin{aligned}
i u_{t}+u_{x^{4}}+\left(8 * \cot ^{2} x-7\right) u & =\epsilon u \circ \dot{\chi}, \quad x \in(0, \pi), t>0, \\
u(x, 0) & =\sin ^{2} x .
\end{aligned}
$$

The exact solution of its deterministic system is $u(x, y, t)=$ $e^{i t} \sin ^{2} x$. 
Figure 4 plots the amplitude $\left|u_{k}^{n}\right|$ for one trajectory. Figure 5 shows the evolution of $\|u\|_{\infty}$ for one trajectory and the average norm over 50 trajectories. We see that the white noise produces stochastic perturbation on the solitary wave and the size of perturbation depends on the size of noise. Figure 5 plots the residuals of discrete charge of one trajectory, which verifies the conservation of discrete charge of the compact schemes. Figure 6 plots the residuals of discrete energy for one trajectory and the average norm over 50 trajectories. The figure tells us that the stochastic noise makes residuals of discrete energy increase linearly over time.

\section{Conclusion}

In this paper, we apply a symplectic scheme in time and a kind of compact difference schemes in space to solve the LSES. The methods are unconditionally stable. Under periodic boundary conditions, they preserve a discrete charge invariant and satisfy a discrete energy transforming law.

\section{Conflict of Interests}

The authors declare that there is no conflict of interests regarding the publication of this paper.

\section{Acknowledgments}

Xiuling Yin is supported by the Director Innovation Foundation of ICMSEC and AMSS, the Foundation of CAS, NNSFC (nos. 91130003 and 11021101). Yanqin Liu is supported by the Natural Science Foundation of Shandong Province (nos. ZR2013AQ005 and BS2013HZ026).

\section{References}

[1] A. Ashyralyev, A. Hanalyev, and P. E. Sobolevskii, "Coercive solvability of the nonlocal boundary value problem for parabolic differential equations," Abstract and Applied Analysis, vol. 6, no. 1, pp. 53-61, 2001.

[2] M. Pašić, "Fite-Wintner-Leighton-type oscillation criteria for second-order differential equations with nonlinear damping," Abstract and Applied Analysis, vol. 2013, Article ID 852180, 10 pages, 2013.

[3] W. M. Abd-Elhameed, E. H. Doha, and Y. H. Youssri, "New wavelets collocation method for solving second-order multipoint boundary value problems using Chebyshev polynomials of third and fourth kinds," Abstract and Applied Analysis, vol. 2013, Article ID 542839, 9 pages, 2013.

[4] K. Ivaz, A. Khastan, and J. J. Nieto, "A numerical method for fuzzy differential equations and hybrid fuzzy differential equations," Abstract and Applied Analysis, vol. 2013, Article ID 735128, 10 pages, 2013.

[5] C. Ashyralyyev, A. Dural, and Y. Sozen, "Finite difference method for the reverse parabolic problem," Abstract and Applied Analysis, vol. 2012, Article ID 294154, 17 pages, 2012.

[6] A. Phillips, Introduction to Quantum Mechanics, John Wiley \& Sons, Chichester, UK, 2003.

[7] G. Milstein and M. Tretyakov, Stochastic Numerics for Mathematical Physics, Kluwer Academic Publishers, Norwell, Mass, USA, 1995.
[8] J. Hong and M.-Z. Qin, "Multisymplecticity of the centred box discretization for Hamiltonian PDEs with $m \geq 2$ space dimensions," Applied Mathematics Letters, vol. 15, no. 8, pp. 1005-1011, 2002.

[9] L. Kong, J. Hong, L. Wang, and F. Fu, "Symplectic integrator for nonlinear high order Schrödinger equation with a trapped term," Journal of Computational and Applied Mathematics, vol. 231, no. 2, pp. 664-679, 2009.

[10] J. Hong, Y. Liu, H. Munthe-Kaas, and A. Zanna, "Globally conservative properties and error estimation of a multi-symplectic scheme for Schrödinger equations with variable coefficients," Applied Numerical Mathematics, vol. 56, no. 6, pp. 814-843, 2006.

[11] J. Hong, X. Liu, and C. Li, "Multi-symplectic Runge-KuttaNyström methods for nonlinear Schrödinger equations with variable coefficients," Journal of Computational Physics, vol. 226, no. 2, pp. 1968-1984, 2007.

[12] Y.-F. Tang, L. Vázquez, F. Zhang, and V. M. Pérez-García, "Symplectic methods for the nonlinear Schrödinger equation," Computers \& Mathematics with Applications, vol. 32, no. 5, pp. 73-83, 1996.

[13] S. Jiang, L. Wang, and J. Hong, "Stochastic multi-symplectic integrator for stochastic nonlinear Schrödinger equation," Communications in Computational Physics, vol. 14, no. 2, pp. 393-411, 2013.

[14] A. Debussche and L. di Menza, "Numerical simulation of focusing stochastic nonlinear Schrödinger equations," Physica D, vol. 162, no. 3-4, pp. 131-154, 2002.

[15] Y. Ma, L. Kong, J. Hong, and Y. Cao, "High-order compact splitting multisymplectic method for the coupled nonlinear Schrödinger equations," Computers \& Mathematics with Applications, vol. 61, no. 2, pp. 319-333, 2011.

[16] T. V. S. Sekhar and B. H. S. Raju, "An efficient higher order compact scheme to capture heat transfer solutions in spherical geometry," Computer Physics Communications, vol. 183, no. 11, pp. 2337-2345, 2012.

[17] A. Mohebbi and Z. Asgari, "Efficient numerical algorithms for the solution of "good" Boussinesq equation in water wave propagation," Computer Physics Communications, vol. 182, no. 12, pp. 2464-2470, 2011.

[18] E. Hairer, C. Lubich, and G. Wanner, Geometric Numerical Integration, vol. 31 of Springer Series in Computational Mathematics, Springer, Berlin, Germany, 2nd edition, 2006.

[19] S. K. Lele, "Compact finite difference schemes with spectral-like resolution," Journal of Computational Physics, vol. 103, no. 1, pp. 16-42, 1992. 


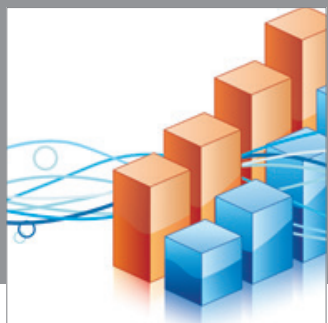

Advances in

Operations Research

mansans

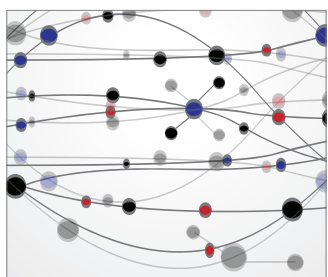

The Scientific World Journal
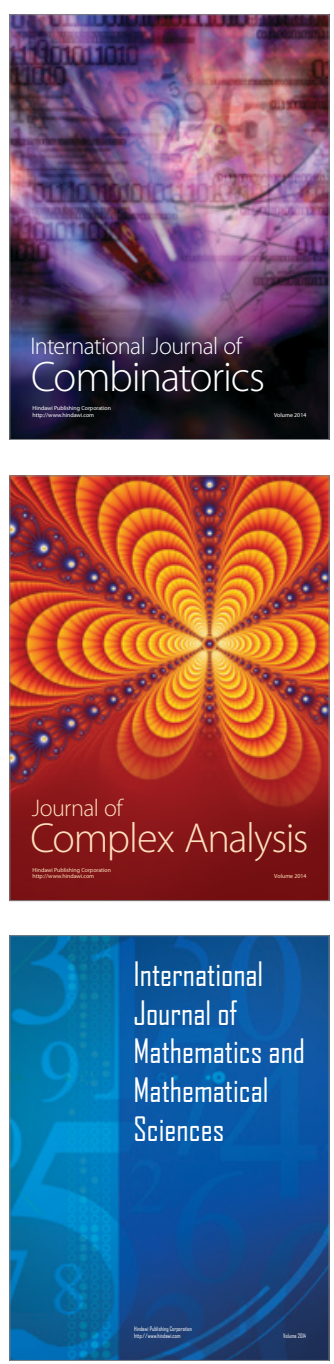
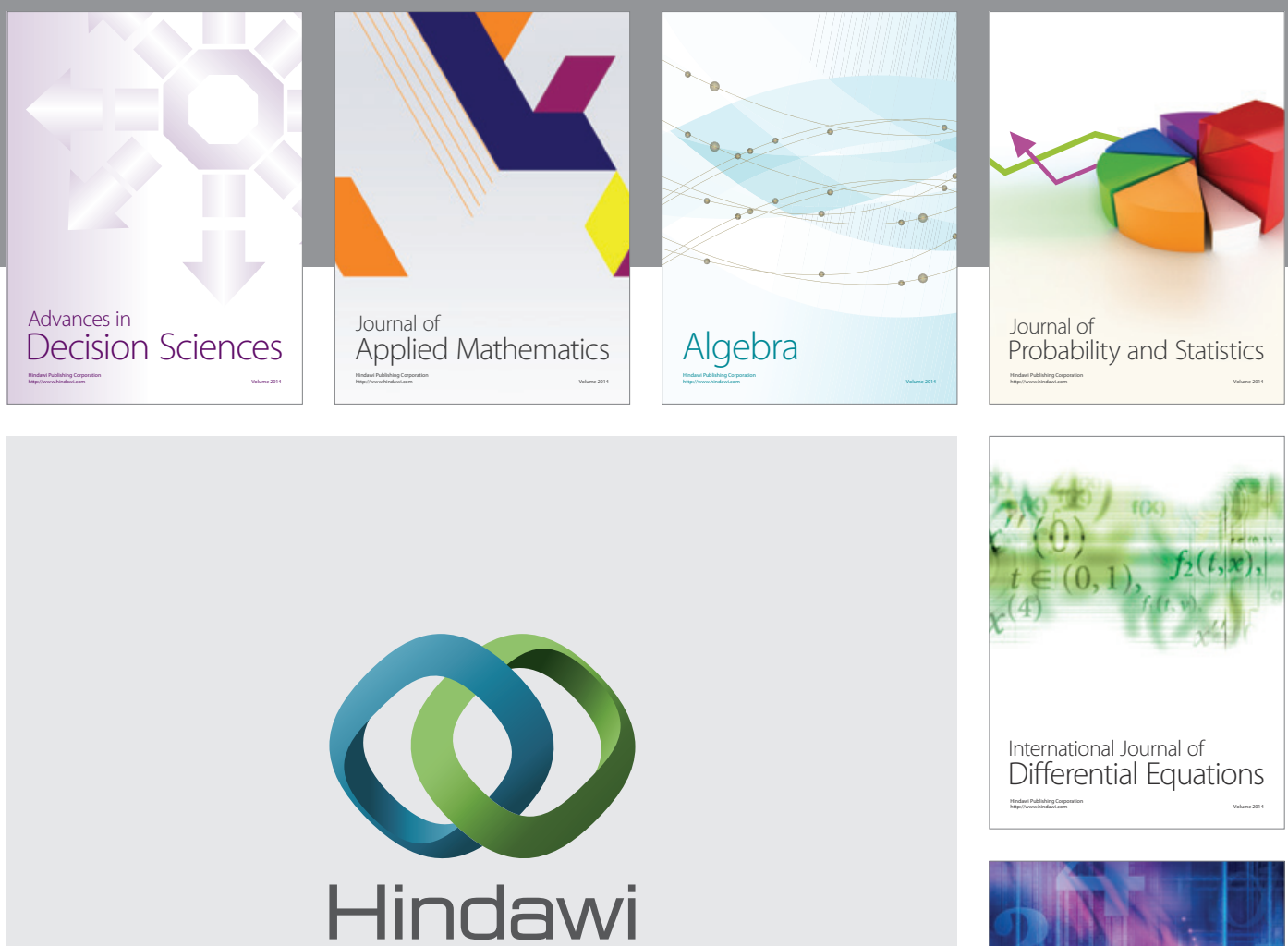

Submit your manuscripts at http://www.hindawi.com
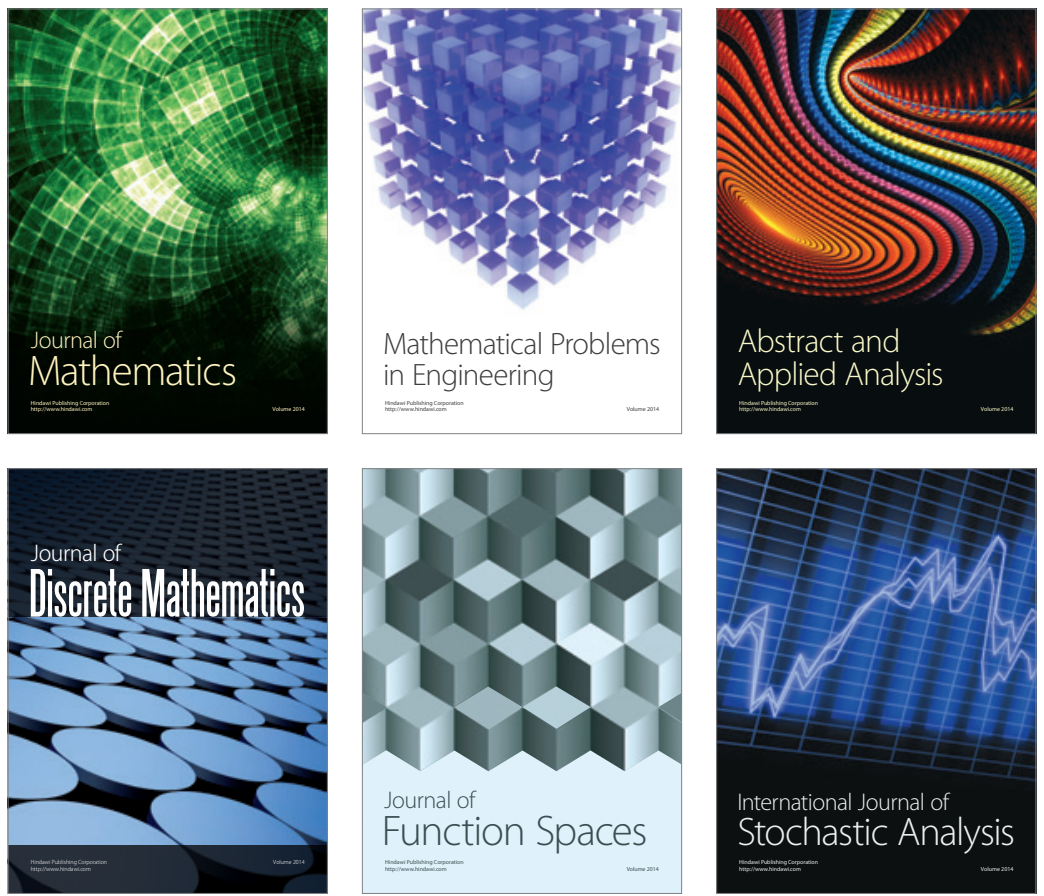

Journal of

Function Spaces

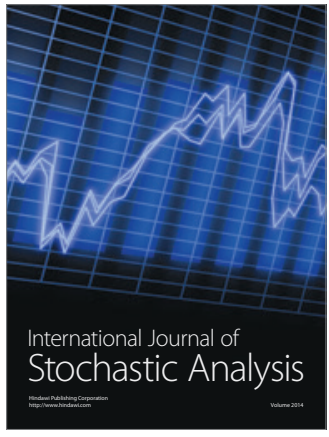

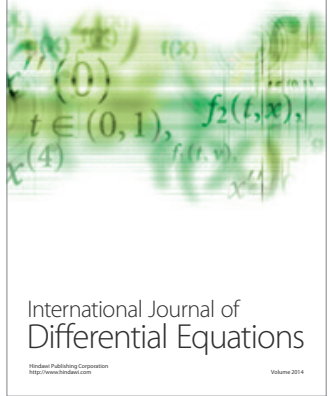
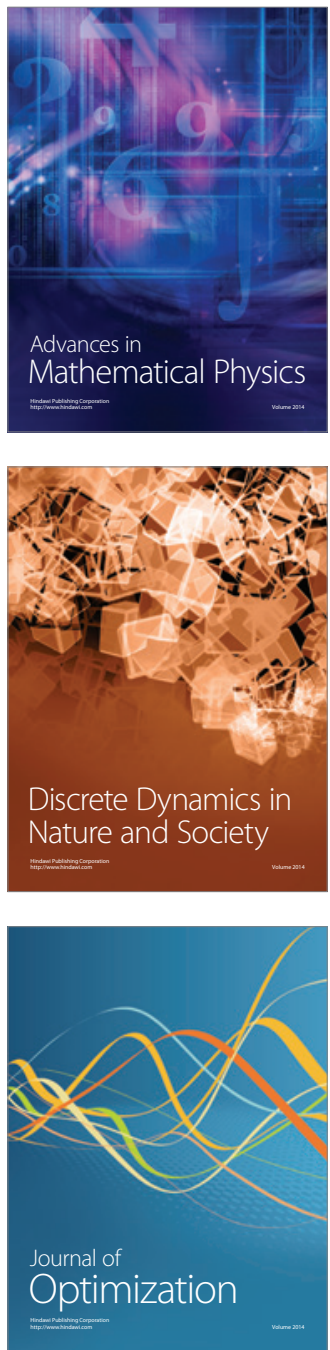